University of Nebraska - Lincoln

DigitalCommons@University of Nebraska - Lincoln

$1-26-2007$

\title{
Tunneling Anisotropic Magnetoresistance Driven by Resonant Surface States: First-Principles Calculations on an Fe(001) Surface
}

\author{
Athanasios N. Chantis \\ Los Alamos National Laboratory, achantis@lanl.gov \\ Kirill D. Belashchenko \\ University of Nebraska-Lincoln, belashchenko@unl.edu \\ Evgeny Y. Tsymbal \\ University of Nebraska-Lincoln, tsymbal@unl.edu \\ Mark van Schilfgaarde \\ Arizona State University, mark.van_schilfgaarde@kcl.ac.uk
}

Follow this and additional works at: https://digitalcommons.unl.edu/cmrafacpub

Part of the Nanoscience and Nanotechnology Commons

Chantis, Athanasios N.; Belashchenko, Kirill D.; Tsymbal, Evgeny Y.; and van Schilfgaarde, Mark, "Tunneling Anisotropic Magnetoresistance Driven by Resonant Surface States: First-Principles Calculations on an Fe(001) Surface" (2007). Faculty Publications from Nebraska Center for Materials and Nanoscience. 79. https://digitalcommons.unl.edu/cmrafacpub/79

This Article is brought to you for free and open access by the Materials and Nanoscience, Nebraska Center for (NCMN) at DigitalCommons@University of Nebraska - Lincoln. It has been accepted for inclusion in Faculty Publications from Nebraska Center for Materials and Nanoscience by an authorized administrator of DigitalCommons@University of Nebraska - Lincoln. 


\title{
Tunneling Anisotropic Magnetoresistance Driven by Resonant Surface States: First-Principles Calculations on an Fe(001) Surface
}

\author{
Athanasios N. Chantis, ${ }^{1, *}$ Kirill D. Belashchenko, ${ }^{2}$ Evgeny Y. Tsymbal, ${ }^{2}$ and Mark van Schilfgaarde ${ }^{1}$ \\ ${ }^{1}$ School of Materials, Arizona State University, Tempe, Arizona 85287, USA \\ ${ }^{2}$ Department of Physics and Astronomy and Nebraska Center for Materials and Nanoscience, University of Nebraska-Lincoln, \\ Lincoln, Nebraska 68588, USA
}

(Received 28 September 2006; published 24 January 2007)

\begin{abstract}
Fully relativistic first-principles calculations of the $\mathrm{Fe}(001)$ surface demonstrate that resonant surface (interface) states may produce sizable tunneling anisotropic magnetoresistance in magnetic tunnel junctions with a single magnetic electrode. The effect is driven by the spin-orbit coupling. It shifts the resonant surface band via the Rashba effect when the magnetization direction changes. We find that spinflip scattering at the interface is controlled not only by the strength of the spin-orbit coupling, but depends strongly on the intrinsic width of the resonant surface states.
\end{abstract}

DOI: 10.1103/PhysRevLett.98.046601

Spin-dependent properties of magnetic surfaces and interfaces have long been a subject of vigorous research. Recent interest is triggered by the advent of magnetoelectronics, a technology aimed at harnessing the electrons spin in data storage and processing. Typical devices utilize heterostructures composed of magnetic and nonmagnetic materials [1]. One approach recently suggested takes advantage of spin-orbit coupling (SOC), where the resistance of a nanostructure is controlled by rotating its magnetization with respect to the interface. For a tunnel junction with only one ferromagnetic electrode, this effect is called tunneling anisotropic magnetoresistance (TAMR) [2-6]. It is different from the usual tunneling magnetoresistance (TMR) observed in magnetic tunnel junctions [7] with two ferromagnetic electrodes when their orientation is switched between the parallel and antiparallel states. It is also different from the usual anisotropic magnetoresistance (AMR) typical for bulk ferromagnets [8] and from ballistic anisotropic magnetoresistance (BAMR) which may occur in ferromagnetic nanocontacts [9]. The TAMR occurs in the tunneling transport regime because SOC makes the electronic structure anisotropic. Practical advantages of TAMR over AMR are (1) the tunneling process filters out a fraction of the electronic phase space, and (2) SOC is usually stronger at interfaces. Both these features result in a larger effect. An advantage of a TAMR device over a conventional magnetic tunnel junction is that only one magnetic interface is necessary.

Experimental demonstrations of TAMR to date have employed diluted magnetic semiconductors (GaAs:Mn) as magnetic electrodes [2-6]. The TAMR effect is due to the anisotropy of the tunneling density of states in the valence band of GaAs induced by SOC [2,3]. It was suggested that metallic alloys, such as $\mathrm{CoPt}$, with large magnetocrystalline anisotropy may be used as electrodes in TAMR devices [10]. TAMR was also observed by scanning tunneling spectroscopy (STS) of thin Fe films on W(110),
PACS numbers: 72.25.Mk, 73.23.-b, 73.40.Gk, 75.47.-m

as the result of a group velocity change from the band splitting induced by SOC [11].

In this Letter, we propose a different route to achieve large TAMR with a metallic electrode. It is well known that many transition-metal surfaces, as well as their interfaces with insulators, exhibit electronic bands that are localized at the surface or interface. If such a band does not mix with bulk states, it is called a surface band. If it mixes weakly with bulk bands, it broadens and becomes a resonant surface band. Interface bands usually contribute strongly to the tunneling current [12-16]. On the other hand, when the magnetization is rotated, these bands shift in the interface Brillouin zone due to the Rashba effect [17] produced by SOC at the interface. For nonmagnetic surfaces, the Rashba effect lifts the spin degeneracy of the surface states [1]; for magnetic surfaces, it leads to an asymmetric shift of the nondegenerate band [18]. The idea pursued in this Letter is to use this Rashba shift in the TAMR device. To illustrate this approach, we choose the $\mathrm{Fe}(001)$ surface because it is one of the most studied transition-metal surfaces supporting a surface minority-spin resonant band at the Fermi level [19]. We show that the change of the magnetization direction both in and out of the surface plane results in a sizable change in the tunneling conductance, which may be observed using STS. We also identify conditions controlling the strength of spin-flip scattering at the interface.

To study TAMR produced by electron tunneling from the $\mathrm{Fe}(001)$ surface through vacuum, we need a counterelectrode for closing the electric circuit. For this purpose, it is convenient to use a metal which does not filter the electrons by the transverse wave vector $\mathbf{k}_{\|}$. A nonmagnetic bcc $\mathrm{Cu}$ electrode has a spin-independent free-electron-like band structure and a featureless surface transmission function [13], which makes it an ideal spin detector, similar to the STS tip in the Tersoff-Hamann theory [20]. The semiinfinite $\mathrm{Fe}$ and $\mathrm{Cu}$ leads are separated by approximately 
$1 \mathrm{~nm}$ of vacuum (6 monolayers of empty spheres). The conductance is calculated using the Landauer-Büttiker approach [21] implemented within the fully-relativistic tight-binding linear muffin-tin orbital (TB-LMTO) method [22]. Charge self-consistency is achieved using scalarrelativistic TB-LMTO calculations for $\mathrm{Fe}$ and $\mathrm{Cu}$ surfaces treated using supercells with 12 metallic monolayers.

The numerical technique is based on the Green's function representation of the (TB-LMTO) method in the atomic spheres approximation [23]. Within the relativistic formulation of the local spin density approximation in which only the spin component of the current density is taken into account [24], inside each atomic sphere we solve the Kohn-Sham Dirac equation [25]. The technique is similar to Refs. [26,27]; the primary difference here is that we use third-order potential functions [28]. The Green's function of the layered system is constructed by a principal-layer technique [29], and the conductance is calculated similar to Ref. [30]. The surface Green's functions of the leads are constructed scalar-relativistically, and therefore the conductance $G$ is represented as a sum of four spin components $G_{\sigma \sigma^{\prime}}=\left(e^{2} / h\right) T_{\sigma \sigma^{\prime}}$ [31], where $T_{\sigma \sigma^{\prime}}$ is the transmission function integrated over $\mathbf{k}_{\|}$(a uniform $200 \times 200$ mesh was used for this integration).

Figs. 1(a)-1(c) show $T_{\sigma \sigma^{\prime}}$ for three magnetization directions. The energy dependence represents the linearresponse conductance in the rigid-band model, which approximately reflects the effects of alloying. It is seen that $T_{\Uparrow}$ exhibits featureless free-electron-like energy dependence. However, $T_{\Downarrow}$ is nonmonotonic and dominates in the energy range between $-125 \mathrm{meV}$ and $25 \mathrm{meV}$. The TAMR ratios $\left(T^{\hat{\mathbf{n}}}-T^{[\mathbf{1 0 0}]}\right) / T^{[\mathbf{1 0 0}]}$ are shown in Fig. 1(d) for both out-of-plane $(\hat{\mathbf{n}}=[001])$ and in-plane $(\hat{\mathbf{n}}=$

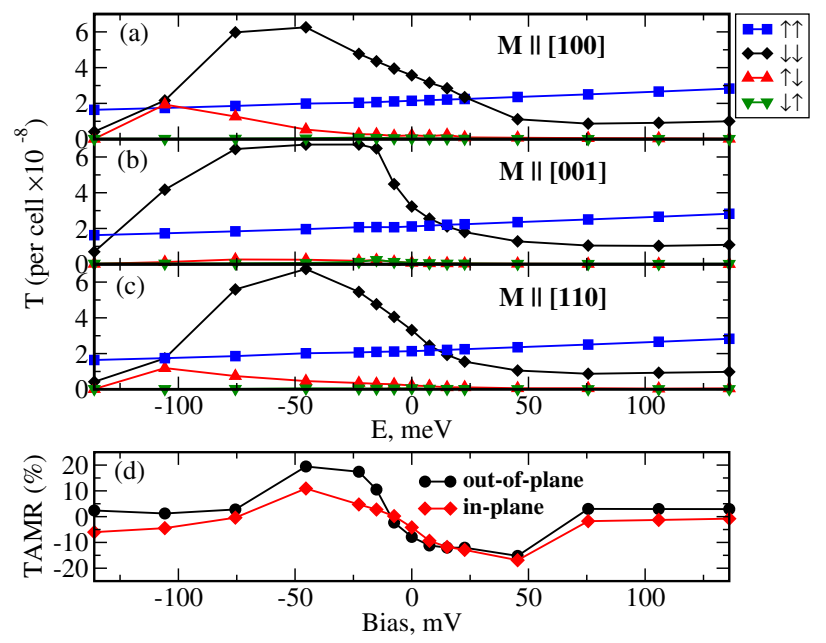

FIG. 1 (color online). (a-c) Spin-resolved integrated transmission $T_{\sigma \sigma^{\prime}}$ for the $\mathrm{Fe}(001)$ surface with a $\mathrm{Cu}$ counterelectrode as a function of energy. Magnetization is along (a) [100], (b) [110], and (c) [001] directions. The Fermi level is at zero energy. (d) In-plane and out-of-plane TAMR as a function of bias voltage.
[110]) magnetization orientations as a function of bias voltage [32]. In both cases, the TAMR has a spectacular change of sign close to the Fermi level and reaches $\pm 15-20 \%$ at the bias voltage of $\mp 50 \mathrm{mV}$.

To explain the origin of large TAMR effect, we focus on energy $-15 \mathrm{meV}$ where the conductance has the strongest spin asymmetry. Figure 2 shows spin- and $\mathbf{k}_{\|}$-resolved surface densities of states (DOS) and tunneling transmission functions. The left six panels correspond to the out-ofplane magnetization $(\hat{\mathbf{n}}=[001])$, and the right half to the in-plane magnetization $(\hat{\mathbf{n}}=[100])$. The resonant surface band is responsible for the bright four-petal-flower features in the minority-spin surface DOS $\left(N_{\downarrow}^{\perp}\right.$ and $\left.N_{\downarrow}^{\|}\right)$and in the minority-spin transmission $\left(T_{\Downarrow}^{\perp}\right.$ and $\left.T_{\Downarrow \downarrow}^{\|}\right)$. This band is dominated by the minority-spin surface states, which mix weakly with bulk bands. A central point is that SOC can strongly enhance this mixing, in both spin-diagonal and spin-mixing components. In particular, consider the surface state lying on the $\bar{\Gamma} \bar{X}$ line with $\mathbf{k}_{\|}=\left(k_{x}, 0\right)$. In the absence of SOC, these eigenstates have definite parity with respect to reflection in the $y=0$ plane. The surface band is even, while the minority-spin bulk band is odd. By symmetry, these states cannot mix, and the surface state remains localized. The SOC does not conserve this parity and mixes the surface state with both minority-spin and majority-spin bulk states. The surface state is thus transformed into a surface resonance. In our case, this occurs at special $\mathbf{k}_{\|}$points. In general, if for a given spin the surface band lies within a gap of bulk bands, the SOC converts this localized surface band into a resonant band.

As is evident from Fig. 2, the surface bands depend on spin orientation (compare $N_{\uparrow}^{\perp}$ to $N_{\uparrow}^{\|}$and $N_{\downarrow}^{\perp}$ to $N_{\downarrow}^{\|}$). This can be attributed to the Rashba effect [17]. The effective spin-orbit shift of electron energy is given by

$$
\Delta \epsilon\left(\mathbf{k}_{\|}\right)=\alpha\left(\hat{\mathbf{z}} \times \mathbf{k}_{\|}\right) \cdot \mathbf{s},
$$

where $\hat{\mathbf{z}}$ is the unit vector normal to the surface. The electron spin $\mathbf{s}$ is aligned with the magnetization M. For $\mathbf{M} \| \hat{\mathbf{z}}$, the Rashba shift is zero throughout the surface Brillouin zone, while for $\mathbf{M} \| \hat{\mathbf{x}}$ it is positive for $k_{y}>0$ and negative for $k_{y}<0$. The asymmetric shift of the surface bands is reflected in the loss of fourfold symmetry in the right six panels of Fig. 2, and, in particular, in the loss of mirror symmetry in $y$. A similar effect was discussed for the $\operatorname{Gd}(0001)$ surface [18].

Comparison of $N_{\uparrow}^{\perp}$ to $N_{\downarrow}^{\perp}$, as well as $N_{\uparrow}^{\|}$to $N_{\downarrow}^{\|}$in Fig. 2 indicates that the admixture of majority-spin states to the surface band is of the order of $1 \%$ (note the difference in scales). However, the spin-flip components of the transmission are quite pronounced. For example, in certain areas of the Brillouin zone, both $T_{\uparrow \downarrow}$ and $T_{\downarrow \uparrow}$ are comparable to the majority-spin component $T_{\Uparrow}$. For $\mathbf{M} \| \hat{\mathbf{z}}$, a portion of the resonant surface band lies close to the $\bar{\Gamma}$ point and adds a large contribution to the minority-spin conductance. When the magnetization is rotated to $\mathbf{M} \| \hat{\mathbf{x}}$, these states 

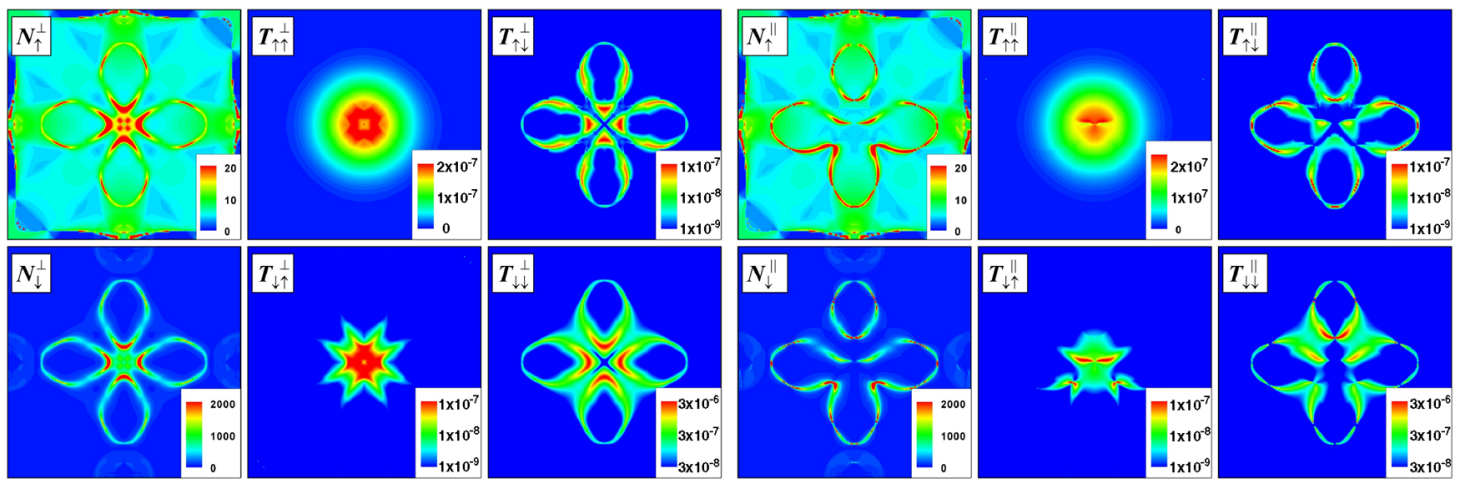

FIG. 2 (color online). Spin components of the DOS $N_{\sigma}$ at the Fe(001) surface monolayer and the transmission functions $T_{\sigma \sigma^{\prime}}$ for the $\mathrm{Fe}(001)$ surface with a $\mathrm{Cu}$ counterelectrode at $15 \mathrm{meV}$ below $E_{F}$. Figures are resolved by $\mathbf{k}_{\|}$with abscissa along [100] and ordinate along [010]. The left six panels correspond to the magnetization normal to the surface (labeled by superscript $\perp$ ), and the right half to the in-plane magnetization aligned along [100] (labeled by superscript II ). Some panels are given in a logarithmic scale.

shift, and the conductance is reduced. This is the origin of the large TAMR effect seen in Fig. 1.

The spin-flip components of the transmission function $T_{\uparrow \downarrow}$ and $T_{\downarrow \uparrow}$ shown in Fig. 1 display a nonmonotonic energy dependence and are generally quite small compared to the spin-conserving components. Surprisingly, for the in-plane magnetization, $T_{\uparrow \downarrow}$ has a pronounced maximum at $E_{F}-$ $0.1 \mathrm{eV}$, just above the bottom of the resonant surface band, which extends to higher energies [Fig. 1(a)]. Here the spinflip and spin-conserving contributions are comparable. Notably, the peak appears only for the in-plane magnetization, and the spin-flip process is strongly asymmetric: $T_{\downarrow \downarrow} \gg T_{\downarrow \downarrow}$. Figure 3 shows $\mathbf{k}_{\|}$-resolved spin-flip transmission function $T_{\uparrow \downarrow}\left(\mathbf{k}_{\|}\right)$for two magnetization directions. The resonant surface bands are seen as four small ellipses along the $\bar{\Gamma} \bar{X}$ lines. All the difference in $T_{\uparrow \downarrow}$ for the two orientations accrues from these four ellipses, which clearly indicates that the large spin-flip conductance is entirely due to the resonant surface states.

To elucidate the origin of the resonant spin-flip transmission, we consider a simple tight-binding model. We assume that there is a single majority band, with surface Green's function $G_{\Uparrow}^{0}$ in the absence of spin-orbit coupling, and a minority surface band $E_{s}\left(\mathbf{k}_{\|}\right)$, broadened by a hybridization with the bulk minority band. The broadening is included through parameter $\gamma_{0}$ that changes the corresponding Green's function into $G_{\Downarrow}^{0}=\left(E-E_{s}\left(\mathbf{k}_{\|}\right)+\right.$ $\left.i \gamma_{0}\right)^{-1}$. The spin-orbit interaction $V_{\mathrm{SO}}$ mixes spin channels resulting in the "dressed" surface Green's function $G_{\sigma \sigma^{\prime}}$.

We apply the Landauer-Büttiker formalism [21] to the case of two coupled spin channels, treating the surface monolayer as a "conductor" and everything else as "leads." We assume that the tunneling probability is small and that the second electrode is nonmagnetic. Then,

$$
T\left(\mathbf{k}_{\|}\right)=\sum_{\sigma \sigma^{\prime}} T_{\sigma \sigma^{\prime}}=4 \sum_{\sigma \sigma^{\prime}} \operatorname{Im} \Sigma_{\sigma}\left|G_{\sigma \sigma^{\prime}}\right|^{2} \operatorname{Im} \Sigma_{v},
$$

where $\Sigma_{\sigma}$ is the spin-dependent self-energy of the surface layer due to interaction with the magnetic electrode, and
$\Sigma_{v}$ the spin-independent self-energy due to interaction with the nonmagnetic electrode through vacuum. Including $V_{\text {SO }}$ through the Dyson equation, we find the spin-flip component of the Green's function

$$
G_{\uparrow \downarrow}=\frac{V_{\mathrm{SO}} G_{\Uparrow}^{0}}{E-E_{s}\left(\mathbf{k}_{\|}\right)+i \gamma_{0}-\left|V_{\mathrm{SO}}\right|^{2} G_{\Uparrow}^{0}} .
$$

Using the identity $\operatorname{Im} G_{\Uparrow}^{0}=\left|G_{\Uparrow}^{0}\right|^{2} \operatorname{Im} \Sigma_{\uparrow}$, and writing $-\left|V_{\text {SO }}\right|^{2} \operatorname{Im} G_{\Uparrow}^{0}$ as $\gamma$, we find for the spin-flip transmission coefficient:

$$
T_{\uparrow \downarrow}\left(\mathbf{k}_{\|}\right) \propto \frac{\gamma}{\left[E-E_{s}\left(\mathbf{k}_{\|}\right)-\Delta\right]^{2}+\left(\gamma_{0}+\gamma\right)^{2}},
$$

where $\Delta=\left|V_{\text {SO }}\right|^{2} \operatorname{Re} G_{\Uparrow}^{0}$ is the shift of the surface band. Finally, we add an energy delta-function and integrate over $\mathbf{k}_{\|}$[33] to obtain

$$
T_{\uparrow \downarrow}(E) \propto N_{s}(E) \frac{\gamma}{\gamma_{0}+\gamma},
$$

where $N_{s}(E)$ is the DOS of the surface band.

The parameters $\gamma$ and $\gamma_{0}$ can be interpreted as broadening of the minority surface band due to the coupling to the majority band (through $V_{\mathrm{SO}}$ ) and to the minority band (through both $V_{\text {SO }}$ and hopping matrix elements), respectively. Equation (5) shows that the spin-flip transmission $T_{\uparrow \downarrow}$ depends on $\gamma / \gamma_{0}$, and is large when $\gamma / \gamma_{0}$ is large. As

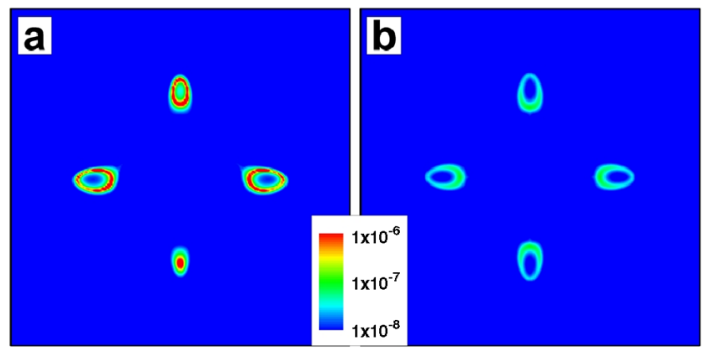

FIG. 3 (color online). Spin-flip transmission $T_{\uparrow \downarrow}$ for magnetization along (a) [100] (in-plane) and (b) [001] (out-of-plane), at $-0.102 \mathrm{meV}$. 
was shown above, along the $\bar{\Gamma} \bar{X}$ lines there is no mixing at all with the minority band when $V_{\mathrm{SO}}=0$. Near the bottom of the interface band, the surface band shrinks to four pockets along these lines (Fig. 3), and hence $\gamma_{0}$ is small for all the surface states at this energy.

The directional dependence of $T_{\uparrow \downarrow}$ (Fig. 3) comes from the relative magnitude of the spin-orbit contributions to $\gamma$ and $\gamma_{0}$. For the perpendicular orientation, $\mathbf{M} \| \hat{\mathbf{z}}, V_{\mathrm{SO}}$ mixes the interface states primarily to minority-spin bulk states, contributing mainly to $\gamma_{0}$. Therefore, $\gamma \ll \gamma_{0}$, and the spin-flip transmission is small. For $\mathbf{M} \| \hat{\mathbf{x}}$, spin-orbit contributions to $\gamma_{0}$ and $\gamma$ are of the same order, and resonant spin-flip transmission sets in. As follows from the Dyson equation, the second spin-flip component $T_{\downarrow \uparrow}$ is smaller than $T_{\uparrow \downarrow}$ by a factor of order $\gamma_{0} / W_{\uparrow}$, where $W_{\uparrow}$ is the bandwidth of the majority bulk band. Thus, the model predicts $T_{\downarrow \uparrow} \ll T_{\uparrow \downarrow}$ in perfect agreement with the detailed calculations, as seen in Fig. 1.

To conclude, we have investigated the electronic structure and tunneling from the $\mathrm{Fe}(001)$ surface to demonstrate that large TAMR may be achieved by utilizing the Rashba shift of interface resonant states. TAMR values of up to $\pm 20 \%$ were predicted for the $\mathrm{Fe}(001)$ surface at small bias voltages; this conclusion may be checked experimentally using STS with a nonmagnetic tip. We also found a spectacular resonant spin-flip transmission near the interface band edge, and showed that spin-flip scattering at the interface depends strongly on the intrinsic broadening of the resonant band. The results are generally applicable to magnetic surfaces and interfaces carrying interface states and suggest that large TAMR may be achieved using metallic electrodes.

The work at UNL was supported by the Nebraska Research Initiative and NSF MRSEC. The work at ASU was supported by DARPA SPINS project, and by ONR.

*Current address: Theoretical Division, Los Alamos National Laboratory, Los Alamos, NM 87545, USA

[1] I. Zutic, J. Fabian, and S. D. Sarma, Rev. Mod. Phys. 76, 323 (2004).

[2] C. Gould, C. Rüster, T. Jungwirth, E. Girgis, G. M. Schott, R. Giraud, K. Brunner, G. Schmidt, and L.W. Molenkamp, Phys. Rev. Lett. 93, 117203 (2004).

[3] C. Rüster, C. Gould, T. Jungwirth, J. Sinova, G. M. Schott, R. Giraud, K. Brunner, G. Schmidt, and L. Molenkamp, Phys. Rev. Lett. 94, 027203 (2005).

[4] A. D. Giddings, M. N. Khalid, T. Jungwirth, J. Wunderlich, S. Yasin, R. P. Campion, K. W. Edmonds, J. Sinova, K. Ito, and K.-Y. Wang et al., Phys. Rev. Lett. 94, 127202 (2005).

[5] H. Saito, S. Yuasa, and K. Ando, Phys. Rev. Lett. 95, 086604 (2005).

[6] R. Giraud, M. Gryglas, L. Thevenard, A. Lemâtre, and G. Faini, Appl. Phys. Lett. 87, 242505 (2005).

[7] E. Y. Tsymbal, O. N. Mryasov, and P. R. LeClair, J. Phys. Condens. Matter 15, R109 (2003).
[8] T. R. McGuire and R. I. Potter, IEEE Trans. Magn. 11, 1018 (1975)

[9] J. Velev, R. F. Sabirianov, S. S. Jaswal, and E. Y. Tsymbal, Phys. Rev. Lett. 94, 127203 (2005).

[10] A. B. Shick, F. Máca, J. Mašek, and T. Jungwirth, Phys. Rev. B 73, 024418 (2006).

[11] M. Bode, S. Heinze, A. Kubetzka, O. Pietzsch, X. Nie, G. Bihlmayer, S. Blugel, and R. Wiesendanger, Phys. Rev. Lett. 89, 237205 (2002).

[12] O. Wunnicke, N. Papanikolaou, R. Zeller, P. H. Dederichs, V. Drchal, and J. Kudrnovský, Phys. Rev. B 65, 064425 (2002).

[13] K. D. Belashchenko, E. Y. Tsymbal, M. van Schilfgaarde, D. A. Stewart, I. I. Oleynik, and S. S. Jaswal, Phys. Rev. B 69, 174408 (2004).

[14] K. D. Belashchenko, E. Y. Tsymbal, I. I. Oleynik, and M. van Schilfgaarde, Phys. Rev. B 71, 224422 (2005).

[15] K. D. Belashchenko, J. Velev, and E. Y. Tsymbal, Phys. Rev. B 72, 140404(R) (2005).

[16] J.P. Velev, K. D. Belashchenko, and E. Y. Tsymbal, Phys. Rev. Lett. 96, 119601 (2006).

[17] E. I. Rashba, Sov. Phys. Solid State 2, 1109 (1960).

[18] O. Krupin, G. Bihlmayer, K. Starke, S. Gorovikov, J. E. Prieto, K. Döbrich, S. Blügel, and G. Kaindl, Phys. Rev. B 71, 201403(R) (2005).

[19] J. A. Stroscio, D. T. Pierce, A. Davies, R. J. Celotta, and M. Weinert, Phys. Rev. Lett. 75, 2960 (1995).

[20] J. Tersoff and D. R. Hamann, Phys. Rev. B 31, 805 (1985).

[21] S. Datta, Electronic transport in mesoscopic systems (Cambridge University Press, Cambridge, England, 1995), Chap. 3.

[22] A. N. Chantis, M. van Schilfgaarde, and V. P. Antropov (to be published)

[23] O. K. Andersen, Phys. Rev. B 12, 3060 (1975).

[24] A. K. Rajagopal and J. Callaway, Phys. Rev. B 7, 1912 (1973).

[25] I. V. Solovyev, A. B. Shick, V.P. Antropov, A. I. Liechtenstein, V. A. Gubanov, and O. K. Andersen, Sov. Phys. Solid State 31, 1285 (1989).

[26] A. B. Shick, V. Drchal, J. Kudrnovsky, and P. Weinberger, Phys. Rev. B 54, 1610 (1996).

[27] I. V. Solovyev, A. I. Liechtenstein, V. A. Gubanov, V.P. Antropov, and O. K. Andersen, Phys. Rev. B 43, 14414 (1991).

[28] O. Gunnarsson, O. Jepsen, and O.K. Andersen, Phys. Rev. B 27, 7144 (1983).

[29] I. Turek, V. Drchal, J. Kudrnovský, M. Šob, and P. Weinberger, Electronic structure of disordered alloys, surfaces and interfaces (Kluwer, Boston, 1997).

[30] J. Kudrnovský, V. Drchal, C. Blaas, P. Weinberger, I. Turek, and P. Bruno, Phys. Rev. B 62, 15084 (2000).

[31] V. Popescu, H. Ebert, N. Papanikolaou, R. Zeller, and P. H. Dederichs, Phys. Rev. B 72, 184427 (2005).

[32] The zero-bias conductance is integrated over energy in the window from $E_{F}$ to $E_{F}+\mathrm{eV}$. This is an excellent approximation appropriate for comparison with STS because the considered voltages are small.

[33] The integration is first performed in the direction normal to the isoenergetic contour, and then along it. Note that the Fermi contour for a 2D surface band is the analog of the Fermi surface for a 3D bulk band. 